1 Hacettepe Journal of Mathematics and Statistics

h Volume 47 (3) (2018), 553-566

\title{
A spectral technique for solving two-dimensional fractional integral equations with weakly singular kernel
}

\author{
Ali H. Bhrawy*, Mohamed A. Abdelkawy ${ }^{\dagger \ddagger}$, Dumitru Baleanu ${ }^{\S}$ and Ahmed
} Z.M. Amin $\|$

\begin{abstract}
This paper adapts a new numerical technique for solving twodimensional fractional integral equations with weakly singular. Using the spectral collocation method, the fractional operators of Legendre and Chebyshev polynomials, and Gauss-quadrature formula, we achieve a reduction of given problems into those of a system of algebraic equations. We apply the reported numerical method to solve several numerical examples in order to test the accuracy and validity. Thus, the novel algorithm is more responsible for solving two-dimensional fractional integral equations with weakly singular.
\end{abstract}

Keywords: Two-dimensional fractional integral equations with weakly singular; Spectral collocation method; Gauss quadrature; Shifted Legendre polynomials; Shifted Chebyshev polynomial

Received : 30.12.2016 Accepted : 10.04.2017 Doi : 10.15672/HJMS.2017.478

\footnotetext{
*Department of Mathematics, Faculty of Science, Beni-Suef University, Beni-Suef, Egypt Email : alibhrawy@yahoo.co.uk

${ }^{\dagger}$ Department of Mathematics and Statistics, College of Science, Al-Imam Mohammad Ibn Saud Islamic University (IMSIU), Riyadh, Saudi Arabia

Email : melkawy@yahoo.com

$\ddagger$ Department of Mathematics, Faculty of Science, Beni-Suef University, Beni-Suef, Egypt

$\S$ Department of Mathematics, Çankaya University, Ankara, Turkey

Email : dumitru@cankaya.edu.tr

IInstitute of Space Sciences, Magurele-Bucharest, Romania

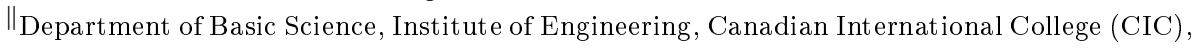
Giza, Egypt

Email : azm.amin@yahoo.com
} 


\section{Introduction}

Integral equations has an important role in many applications in various areas, including mathematical chemistry, electrochemistry, physics, semi-conductors, seismology, scattering theory, heat conduction, fluid flow, metallurgy, population dynamics and chemical reaction [1]-[6]. Fractional calculus provides high accuracy in modeling many engineering and physical phenomena, such as anomalous transport [7], economics [8], bioengineering [9] and others [10]-[15]. As the increasing of employing fractional differential and integral equations in many social and scientific fields, the main challenge we confront is that obtaining solutions for them. Unfortunately, for most of these fractional problems, no one able to achieve analytic solutions for such problems. Therefore, the creation, improvement and development of numerical methods for solving fractional problems have received considerable attention in recent years [16]-[23].

In recent three decades, spectral methods (see [24]-[33]) are often efficient and highly accurate numerical schemes when compared with other local schemes. The main characteristic of the spectral methods is the exponential rate of convergence. In addition, they also have a high level of accuracy. From the overview of methodology, the spectral method has been classified into four kinds namely collocation [34]-[40], tau [41, 42], Galerkin [43] and Petrov-Galerkin [45] methods. In spectral collocation method, we enforce the numerical solution to almost satisfy the mentioned problem as closely as possible. Thus, the residuals may be allowing to be zero at specific collocation nodes [46, 47].

The main aim is to develop a numerical method for solving two-dimensional fractional integral equations. Utilizing the spectral collocation method with some properties of the left-sided mixed Riemann-Liouville integral of order $\nu$ and combination between shifted Legendre polynomial and shifted Chebyshev polynomial, we reduce such problems into a system of algebraic equations which greatly simplifies the problem. Finally, we implement this technique to achieve approximate solutions for numerous examples for the present problems to ensure that the proposed spectral algorithm is accurate and efficient compared with alternative methods.

The outline of the paper is arranged as follows. We present few properties of fractional calculus, shifted Legendre polynomial and shifted Chebyshev polynomials in the next section. In Section 3, we introduce the novel method for solving two-dimensional fractional integral equations with weakly singular. Numerical examples and simulations are presented in Section 4 to show the accuracy and effectiveness of the proposed underlying method. In the last Section, some observations and conclusions are introduced.

\section{Mathematical preliminaries}

\subsection{Fractional calculus.}

2.1. Definition. The operator $J^{\mu}$ of Riemann-Liouville fractional integral has the form $[15,21]$

$$
\begin{aligned}
J^{\mu} f(x) & =\frac{1}{\Gamma(\mu)} \int_{0}^{x}(x-\zeta)^{\mu-1} f(\zeta) d \zeta, \quad x>0, \\
J^{0} f(x) & =f(x)
\end{aligned}
$$

where

$$
\Gamma(\mu)=\int_{0}^{\infty} x^{\mu-1} e^{-x} d x
$$


and $\mu>0$. The operator $J^{\mu}$ fulfills the following properties [15, 21]

$$
\begin{aligned}
& J^{\nu} J^{\mu} f(x)=J^{\nu+\mu} f(x), \\
& J^{\nu} J^{\mu} f(x)=J^{\mu} J^{\nu} f(x), \\
& J^{\mu} x^{\beta}=\frac{\Gamma(\beta+1)}{\Gamma(\beta+1+\mu)} x^{\beta+\mu} .
\end{aligned}
$$

2.1. Definition. [15, 21] The next equation define Riemann-Liouville fractional derivative $D^{\mu}$ of order $\mu$

$$
D^{\mu} f(x)=\frac{1}{\Gamma(m-\mu)} \frac{d^{m}}{d x^{m}}\left(\int_{0}^{x}(x-t)^{m-\mu-1} f(t) d t\right), \quad m-1<\mu \leq m, x>0,
$$

where $m$ is the ceiling function of $\mu>0$.

2.2. Definition. Let $\nu=\left(\nu_{1}, \nu_{2}\right) \in(0, \infty) \times(0, \infty)$, and $\alpha=(0,0)$, the left-sided mixed Riemann-Liouville integral of order $\nu$ of $u$ is defined as [11, 12]

$$
J_{\alpha}^{\nu} u(x, t)=\frac{1}{\Gamma\left(\nu_{1}\right) \Gamma\left(\nu_{2}\right)} \int_{0}^{x} \int_{0}^{t}(x-y)^{\nu_{1}-1}(t-z)^{\nu_{2}-1} u(y, z) d z d y,
$$

the properties of left-sided mixed Riemann-Liouville integral:

$$
\begin{aligned}
& J_{\alpha}^{\alpha} u(x, t)=u(x, t), \quad\left(J_{\alpha}^{\nu} u\right)(x, 0)=\left(J_{\alpha}^{\nu} u\right)(0, t)=0, \\
& J_{\alpha}^{\mu} u(x, t)=\int_{0}^{x} \int_{0}^{t} u(x, t) d y d z, \quad \text { where } \quad \mu=(1,1), \\
& J_{\alpha}^{\nu} x^{\lambda} t^{\omega}=\frac{\Gamma(\lambda+1) \Gamma(\omega+1)}{\Gamma\left(\lambda+\nu_{1}+1\right) \Gamma\left(\omega+\nu_{2}+1\right)} x^{\lambda+\nu_{1}} t^{\omega+\nu_{2}} .
\end{aligned}
$$

2.2. Shifted Legendre Gauss-Lobatto interpolation. In this subsection, we list some approximation results for the shifted Legendre Gauss-Lobatto (SL-GL) interpolation. The Legendre polynomials $P_{k}(x)(k=0,1 \ldots)$ satisfy the Rodrigue's formula

$$
P_{k}(x)=\frac{(-1)^{k}}{2^{k} k !} D^{k}\left(\left(1-x^{2}\right)^{k}\right) .
$$

Accordingly, $P_{k}^{(p)}(x)$ (the $p$ th derivative of $\left.P_{k}(x)\right)[48,49,50]$ is given by

$$
P_{k}^{(p)}(x)=\sum_{i=0(i+k=\text { even })}^{k-p} C_{p}(k, i) P_{i}(x),
$$

where

$$
C_{p}(k, i)=\frac{2^{p-1}(2 i+1) \Gamma\left(\frac{p+k-i}{2}\right) \Gamma\left(\frac{p+k+i+1}{2}\right)}{\Gamma(p) \Gamma\left(\frac{2-p+k-i}{2}\right) \Gamma\left(\frac{3-p+k+i}{2}\right)} .
$$

Next, denoting by $\|u\|$ and $(u, v)$ the norm and inner product of space $L^{2}[-1,1]$. The set of $P_{k}(x)$ is a complete orthogonal system in $L^{2}[-1,1]$

$$
\left(P_{j}(x), P_{k}(x)\right)=\int_{-1}^{1} P_{j}(x) P_{k}(x) d x=h_{k} \delta_{j k},
$$


where $h_{i}=\frac{2}{2 i+1}$ and $\delta_{j k}$ is the Dirac function. Thus for any $v \in L^{2}[-1,1]$,

$$
v(x)=\sum_{i=0}^{\infty} a_{i} P_{i}(x), \quad a_{i}=\frac{1}{h_{i}} \int_{-1}^{1} v(x) P_{i}(x) d x .
$$

Let $S_{N}[-1,1]$ be the set of all polynomials of degree at most $N(N \geq 0)$. Thus, for any $\varphi \in S_{2 N-1}[-1,1]$ we have

$$
\int_{-1}^{1} \varphi(x) d x=\sum_{i=0}^{N} \varpi_{N, i} \varphi\left(x_{N, i}\right)
$$

where $x_{N, k}(0 \leq k \leq N)$ and $\varpi_{N, k}(0 \leq k \leq N)$ are denoted to the nodes and Christoffel numbers of Legendre Gauss-Lobatto (L-GL) interpolation on the classical interval [-1, 1], respectively. The norm and discrete inner product are defined as

$$
\|u\|_{N}=(u, v)_{N}^{\frac{1}{2}}, \quad(u, v)_{N}=\sum_{j=0}^{N} u\left(x_{N, j}\right) v\left(x_{N, j}\right) \varpi_{N, j} .
$$

Let us denote by $P_{L, k}(x)$ the shifted Legendre polynomials which defined on the interval $[0, L]$. These polynomials can be engendered from the recurrence relation:

$$
(k+1) P_{L, k+1}(x)=(2 k+1)\left(\frac{2 x}{L}-1\right) P_{L, k}(x)-k P_{L, k-1}(x), \quad k=1,2, \cdots .
$$

The analytic form of $P_{L, i}(x)$ may be written as

$$
P_{L, j}(x)=\sum_{k=0}^{j}(-1)^{j+k} \frac{(j+k) !}{(j-k) !(k !)^{2} L^{k}} x^{k} .
$$

The orthogonality condition is

$$
\int_{0}^{L} P_{L, j}(x) P_{L, k}(x) d x=h_{k}^{L} \delta_{j k}
$$

and $h_{k}^{L}=\frac{L}{2 k+1}$.

If function $u(t) \in L^{2}[0, L]$. Then one can express it by means of $P_{L, i}(t)$ as

$$
u(t)=\sum_{i=0}^{\infty} c_{i} P_{L, i}(t)
$$

where $c_{i}$ is given by

$$
c_{i}=\frac{1}{h_{i}^{L}} \int_{0}^{L} u(t) P_{L, i}(t) d x, \quad i=0,1,2, \cdots .
$$

In the approximation $u(x)$ may be expanded as

$$
u_{N}(t) \simeq \sum_{i=0}^{N} c_{i} P_{L, i}(t) .
$$


2.3. Shifted Chebyshev Gauss-Radau interpolation. The Chebyshev polynomials defined on $[-1,1]$ fulfill the following recurrence formula

$$
T_{i+1}(t)=2 t T_{i}(t)-T_{i-1}(t), i=1,2, \ldots
$$

Also

(2.20) $\quad T_{k}( \pm 1)=( \pm 1)^{k}, \quad T_{k}(-t)=(-1)^{k} T_{k}(t)$.

Let $w^{c}(t)=\frac{1}{\sqrt{1-t^{2}}}$, the we introduce the following norm and inner product of the weighted space $L_{w^{c}}^{2}$ as

$$
\|u\|_{w^{c}}=(u, u)_{w^{c}}^{\frac{1}{2}}, \quad(u, v)_{w^{c}}=\int_{-1}^{1} u(t) v(t) w^{c}(t) d t .
$$

The set of Chebyshev polynomials satisfies:

$$
\int_{-1}^{1}\left(1-t^{2}\right)^{-\frac{1}{2}} T_{k}(t) T_{j}(t) d x=\delta_{k j} h_{k}^{c}
$$

where

$$
h_{k}^{c}= \begin{cases}\pi, & k=0 \\ \frac{\pi}{2}, & k \geq 1\end{cases}
$$

Now, we define the following norm and discrete inner product

$$
\|u\|_{w^{c}}=(u, u)_{w^{c}}^{\frac{1}{2}}, \quad(u, v)_{w^{c}}=\sum_{j=0}^{N} u\left(t_{N, j}\right) v\left(t_{N, j}\right) \varpi_{N, j}^{c} .
$$

Let us denote by $T_{T, n}(t)$ the shifted Chebyshev polynomials which defined on the interval $[0, T]$. The analytic form of $T_{T, n}(t)$ is obtained from

$$
T_{T, n}(t)=n \sum_{k=0}^{n}(-1)^{n-k} \frac{(n+k-1) ! 2^{2 k}}{(n-k) !(2 k) ! T^{k}} t^{k}
$$

where $T_{T, n}(0)=(-1)^{n}$ and $T_{T, n}(T)=1$.

The expression of the orthogonality condition read as

$$
\int_{0}^{L} T_{T, m}(t) T_{T, n}(x) w_{T}(x) d x=\delta_{m n}{ }^{c} h_{n}^{T},
$$

where $w_{T}(t)=\frac{1}{\sqrt{T t-t^{2}}}$ and ${ }^{c} h_{n}^{T}=\frac{c_{n}}{2} \pi$, with $c_{0}=2, c_{i}=1, i \geq 1$.

As in the previous subsection, if $u(t) \in L_{w_{T}(t)}^{2}[0, T]$. Then one can express it by means of $T_{T, i}(t)$ as

$$
u(t)=\sum_{j=0}^{\infty} a_{j} T_{T, j}(t)
$$

where

$$
a_{j}=\frac{1}{{ }^{c} h_{j}^{T}} \int_{0}^{T} u(t) T_{T, j}(t) w_{T}(t) d t, \quad j=0,1,2, \cdots .
$$




\section{Two-dimensional fractional integral equation with weakly sin- gular}

Below we utilized the spectral collocation method to solve the two-dimensional fractional integral equation with weakly singular in the following form

$$
u(x, t)=\int_{0}^{x} \int_{0}^{t}(x-y)^{\nu_{1}-1}(t-\tau)^{\nu_{2}-1} u(y, \tau) d y d \tau+f(x, t),
$$

where $g(x, t)$ denotes a given function, and $u(x, t)$ represents the unknown function. Adopting (2.4), the integral equation (3.1) is transformed to the fractional integral equation as:

$$
u(x, t)=\Gamma\left(\nu_{1}\right) \Gamma\left(\nu_{2}\right) J_{\alpha}^{\nu} u(x, t)+f(x, t) .
$$

The use of the spectral collocation method based on shifted Legendre and shifted Chebyshev polynomials requires to approximate $u(x, t)$ as

$$
u_{N, M}(x, t)=\sum_{i=0}^{M} \sum_{j=0}^{N} a_{i j} P_{L, j}(x) T_{\tau, i}(t) .
$$

In virtue of (2.7), (2.15), and (2.24), we can express the fractional integration of order $\nu$ of any shifted Legendre polynomial and shifted Chebyshev polynomial $P_{L, j}(x) T_{\tau, i}(t)$ as

$$
\begin{aligned}
J^{\mu}\left(P_{L, j}(x) T_{\tau, i}(t)\right)= & P_{L, j}^{\nu_{1}}(x) T_{\tau, i}^{\nu_{2}}(t) \\
= & \sum_{k=0}^{j}(-1)^{j+k} \frac{(j+k) !}{\left(k+\nu_{1}\right) !(j-k) !(k !) L^{k}} \times \\
& i \sum_{\lambda=0}^{i}(-1)^{i-\lambda} \frac{\lambda !(i+\lambda-1) ! 2^{2 \lambda}}{\left(\lambda+\nu_{2}\right) !(i-\lambda) !(2 \lambda) ! L_{2}^{\lambda}} x^{k+\nu_{1}} t^{\lambda+\nu_{2}},
\end{aligned}
$$

therefore, easily we can write

$$
J^{\nu} u_{N, M}(x, t)=\sum_{i=0}^{M} \sum_{j=0}^{N} a_{i j} P_{L, j}^{\left(\nu_{1}\right)}(x) T_{\tau, i}^{\left(\nu_{2}\right)}(t)
$$

Therefore, adopting (3.2) with (3.5), we can write (3.1) in the form:

$$
\sum_{i=0}^{M} \sum_{j=0}^{N} a_{i j} P_{L, j}(x) T_{\tau, i}(t)=\Gamma\left(\nu_{1}\right) \Gamma\left(\nu_{2}\right) \sum_{i=0}^{M} \sum_{j=0}^{N} a_{i j} P_{L, j}^{\left(\nu_{1}\right)}(x) T_{\tau, i}^{\left(\nu_{2}\right)}(t)+f(x, t) .
$$

Now we utilized the spectral collocation method by setting the residual of the previous equation to be zero at the $(N+1)(M+1)$ collocation points, namely:

$$
\sum_{i=0}^{M} \sum_{j=0}^{N} a_{i j} P_{L, j}\left(x_{L, N, k}\right) T_{\tau, i}\left(t_{\tau, M, m}\right)=\psi(k, m), k=0,1, \ldots, N, m=0,1, \ldots, M
$$

where $\psi(k, m)=\Gamma\left(\nu_{1}\right) \Gamma\left(\nu_{2}\right) \sum_{i=0}^{M} \sum_{j=0}^{N} a_{i j} P_{L, j}^{\left(\nu_{1}\right)}\left(x_{L, N, k}\right) T_{\tau, i}^{\left(\nu_{2}\right)}\left(t_{\tau, M, m}\right)+f\left(x_{L, N, k}, t_{\tau, M, m}\right)$.

Thus, we get:

$$
\left(\mathbf{P} \mathbf{T}+\Gamma\left(\nu_{1}\right) \Gamma\left(\nu_{2}\right) \mathbf{P}^{\left(\nu_{1}\right)} \mathbf{T}^{\left(\nu_{2}\right)}\right) \mathbf{A}=\mathbf{F},
$$




$$
\begin{aligned}
& \mathbf{P}^{\left(\nu_{1}\right)}=\left(\begin{array}{cccc}
P_{L, 0}^{\left(\nu_{1}\right)}\left(x_{L, N, 0}\right) & P_{L, 1}^{\left(\nu_{1}\right)}\left(x_{L, N, 0}\right) & \ldots & P_{L, N}^{\left(\nu_{1}\right)}\left(x_{L, N, 0}\right) \\
P_{L, 0}^{\left(\nu_{1}\right)}\left(x_{L, N, 1}\right) & P_{L, 1}^{\left(\nu_{1}\right)}\left(x_{L, N, 1}\right) & \ldots & P_{L, N}^{\left(\nu_{1}\right)}\left(x_{L, N, 1}\right) \\
\vdots & \vdots & \ddots & \vdots \\
P_{L, 0}^{\left(\nu_{1}\right)}\left(x_{L, N, i}\right) & P_{L, 1}^{\left(\nu_{1}\right)}\left(x_{L, N, i}\right) & \ldots & P_{L, N}^{\left(\nu_{1}\right)}\left(x_{L, N, i}\right) \\
\vdots & \vdots & \ddots & \vdots \\
P_{L, 0}^{\left(\nu_{1}\right)}\left(x_{L, N, N-1}\right) & P_{L, 1}^{(\nu)}\left(x_{L, N, N-1}\right) & \ldots & P_{L, N}^{\left(\nu_{1}\right)}\left(x_{L, N, N-1}\right) \\
P_{L, 0}^{\left(\nu_{1}\right)}\left(x_{L, N, N}\right) & P_{L, 1}^{\left(\nu_{1}\right)}\left(x_{L, N, N}\right) & \ldots & P_{L, N}^{\left(\nu_{1}\right)}\left(x_{L, N, N}\right)
\end{array}\right) \\
& \mathbf{T}^{\left(\nu_{2}\right)}=\left(\begin{array}{cccc}
T_{\tau, 0}^{\left(\nu_{2}\right)}\left(t_{\tau, M, 0}\right) & T_{\tau, 1}^{\left(\nu_{2}\right)}\left(t_{\tau, M, 0}\right) & \ldots & T_{\tau, M}^{\left(\nu_{2}\right)}\left(x_{\tau, M, 0}\right) \\
T_{\tau, 0}^{\left(\nu_{2}\right)}\left(t_{\tau, M, 1}\right) & T_{\tau, 1}^{\left(\nu_{2}\right)}\left(t_{\tau, M, 1}\right) & \ldots & T_{\tau, M}^{\left(\nu_{2}\right)}\left(t_{\tau, M, 1}\right) \\
\vdots & \vdots & \ddots & \vdots \\
T_{\tau, 0}^{\left(\nu_{2}\right)}\left(t_{\tau, M, i}\right) & T_{\tau, 1}^{\left(\nu_{2}\right)}\left(t_{\tau, M, i}\right) & \ldots & T_{\tau, N}^{\left(\nu_{2}\right)}\left(t_{\tau, M, i}\right) \\
\vdots & \vdots & \ddots & \vdots \\
T_{\tau, 0}^{\left(\nu_{2}\right)}\left(t_{\tau, M, M-1}\right) & T_{\tau, 1}^{\left(\nu_{2}\right)}\left(t_{\tau, M, M-1}\right) & \ldots & T_{\tau, N}^{\left(\nu_{2}\right)}\left(t_{\tau, M, M-1}\right) \\
T_{\tau, 0}^{\left(\nu_{2}\right)}\left(t_{\tau, M, M}\right) & T_{\tau, 1}^{\left(\nu_{2}\right)}\left(t_{\tau, M, M}\right) & \ldots & T_{\tau, N}^{\left(\nu_{2}\right)}\left(t_{\tau, M, M}\right)
\end{array}\right) \\
& \mathbf{A}=\left(\begin{array}{cccc}
a_{00} & a_{01} & \ldots & a_{0 N} \\
a_{10} & a_{11} & \ldots & a_{1 N} \\
\vdots & \vdots & \ddots & \vdots \\
a_{M 0} & a_{M 1} & \ldots & a_{M N}
\end{array}\right) \\
& \mathbf{F}=\left(\begin{array}{cccc}
f_{00} & f_{01} & \ldots & f_{0 N} \\
f_{10} & f_{11} & \ldots & f_{1 N} \\
\vdots & \vdots & \ddots & \vdots \\
f_{M 0} & f_{M 1} & \ldots & f_{M N}
\end{array}\right)
\end{aligned}
$$

where $F=f\left(x_{L, N, k}, t_{\tau, M, m}\right)$. We recall that we can easily solve the obtained system of algebraic equations.

\section{Numerical results}

To demonstrate the effectiveness of the proposed method in the present paper, three test examples are carried out in this section. The comparisons of the numerical results detect that the present algorithm is very appropriate and effective. The numerical results obtained in this paper are computing by means of mathematica program. The absolute error $(\mathrm{AE})$ is defined as

$$
E(x)=\left|u(x)-u_{N}(x)\right|,
$$

where $u(x)$ and $u_{N}(x)$ are the exact and the approximate solutions at the point $x$, respectively. While, the maximum absolute errors (MAE) is given by

$$
\operatorname{MAE}=\operatorname{Max}\{E(x)\} .
$$

1. Example. Consider the following problem

$$
u(x, t)=f(x, t)+\int_{0}^{x} \int_{0}^{t}(x-y)^{\frac{1}{3}}(t-\tau)^{-\frac{1}{3}} u(y, \tau) d \tau d y
$$

where $f(x, t)$ is a given source term such that the exact solution is $u(x, t)=x^{2}\left(t^{2}-t\right)$. 
Table 1. AEs for Example 1.

\begin{tabular}{|c|c|c|c|c|}
\hline$(x, t)$ & $N=M=2$ & $N=M=4$ & $N=M=6$ & $N=M=8$ \\
\hline$(0,0)$ & $1.39 \times 10^{-16}$ & $6.92 \times 10^{-17}$ & $3.00 \times 10^{-25}$ & $1.58 \times 10^{-39}$ \\
$(0.1,0.1)$ & $1.28 \times 10^{-16}$ & $1.53 \times 10^{-16}$ & $2.11 \times 10^{-26}$ & $1.39 \times 10^{-17}$ \\
$(0.2,0.2)$ & $1.25 \times 10^{-16}$ & $9.71 \times 10^{-17}$ & $2.08 \times 10^{-17}$ & $6.94 \times 10^{-18}$ \\
$(0.3,0.3)$ & $1.53 \times 10^{-16}$ & $1.68 \times 10^{-16}$ & $1.39 \times 10^{-17}$ & $1.39 \times 10^{-17}$ \\
$(0.4,0.4)$ & $1.94 \times 10^{-16}$ & $2.36 \times 10^{-16}$ & $1.39 \times 10^{-17}$ & $1.39 \times 10^{-17}$ \\
$(0.5,0.5)$ & $2.08 \times 10^{-16}$ & $2.78 \times 10^{-16}$ & $1.39 \times 10^{-17}$ & $6.94 \times 10^{-18}$ \\
$(0.6,0.6)$ & $1.67 \times 10^{-16}$ & $2.50 \times 10^{-16}$ & $2.78 \times 10^{-17}$ & $2.78 \times 10^{-17}$ \\
$(0.7,0.7)$ & $2.50 \times 10^{-16}$ & $3.47 \times 10^{-16}$ & $1.39 \times 10^{-17}$ & $1.39 \times 10^{-17}$ \\
$(0.8,0.8)$ & $2.71 \times 10^{-16}$ & $3.33 \times 10^{-16}$ & $2.34 \times 10^{-25}$ & $3.37 \times 10^{-39}$ \\
$(0.9,0.9)$ & $2.81 \times 10^{-16}$ & $3.33 \times 10^{-16}$ & $1.38 \times 10^{-24}$ & $3.63 \times 10^{-39}$ \\
\hline
\end{tabular}

Table 1 lists the results obtained by the technique explained in Section 3 in terms of the AEs with different choices of $N$ and $M$. We observe very highly accurate results are listed in Table 1. The space-graph of the AE $E(x, t)$ of Example 1 for $N=M=8$, is displayed in Fig. 1.

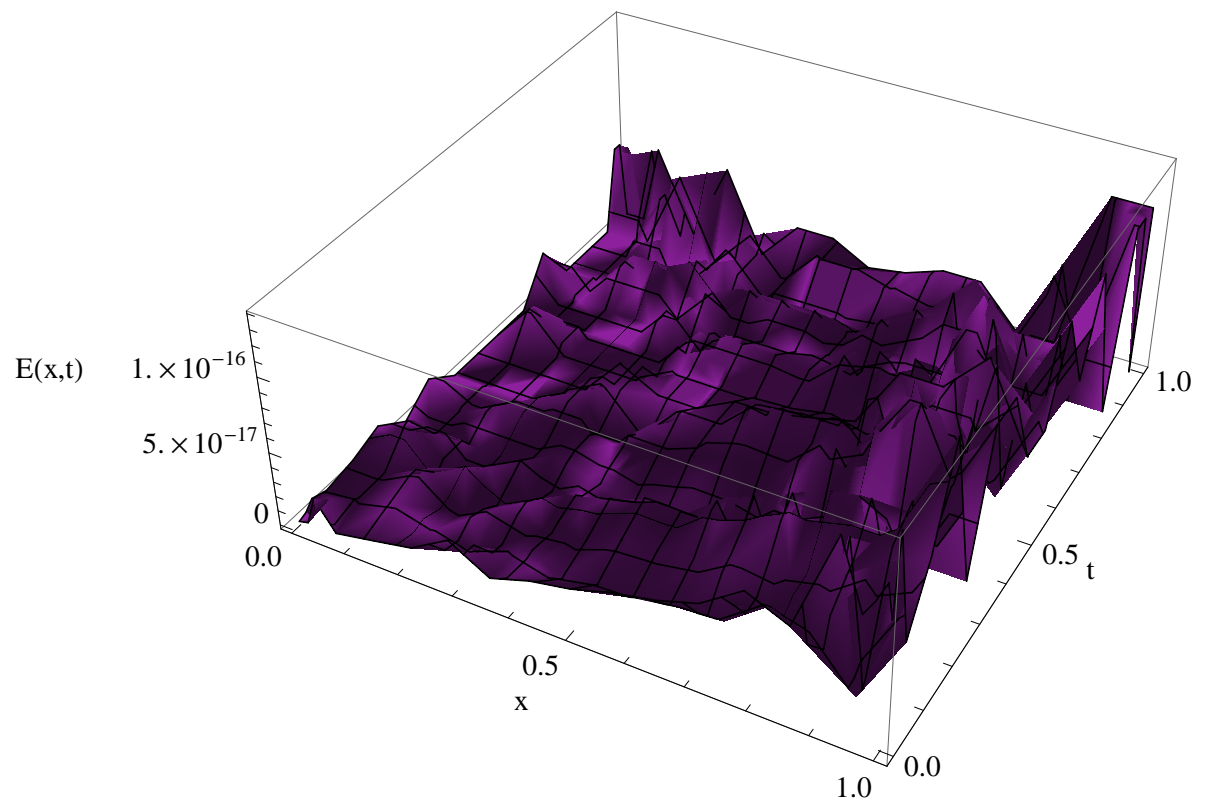

Figure 1. Space-graph of the AE related to Example 1 for $N=M=8$. 
2. Example. Let us analyze the following problem

$$
u(x, t)=f(x, t)+\int_{0}^{x} \int_{0}^{t}(x-y)^{\frac{1}{2}}(t-\tau)^{\frac{-1}{2}} u(y, \tau) d \tau d y
$$

where $f(x, t)$ is extracted from the exact solution $u(x, t)=x^{2}-t$.

To test the convergence rate of our method, we list absolute errors in Table 2. The results show that we obtain a numerical solution with better accuracy with far fewer nodes. Also, we can observe that our numerical solutions coincide closely with the exact ones. Figs. 2, 3 show the space-graphs of AE $E(x, t)$ of Example 1 for $N=M=4$ and $N=M=8$, respectively. In the case of $N=M=4$, the absolute error curve in $t$ - and $x$-direction of Example 2 are shown in Figs. 4 and 5, respectively.

Table 2. AEs for Example 2.

\begin{tabular}{|c|c|c|c|c|}
\hline$(x, t)$ & $N=M=2$ & $N=M=4$ & $N=M=6$ & $N=M=8$ \\
\hline$(0,0)$ & $5.55 \times 10^{-17}$ & $2.38 \times 10^{-16}$ & $3.34 \times 10^{-31}$ & $6.38 \times 10^{-30}$ \\
$(0.1,0.1)$ & $1.11 \times 10^{-16}$ & $1.02 \times 10^{-16}$ & $1.39 \times 10^{-29}$ & $5.78 .39 \times 10^{-30}$ \\
$(0.2,0.2)$ & $5.55 \times 10^{-17}$ & $2.07 \times 10^{-16}$ & $5.55 \times 10^{-17}$ & $5.55 \times 10^{-17}$ \\
$(0.3,0.3)$ & 0 & $1.49 \times 10^{-16}$ & $3.69 \times 10^{-28}$ & $1.93 \times 10^{-30}$ \\
$(0.4,0.4)$ & 0 & $2.03 \times 10^{-16}$ & $6.64 \times 10^{-28}$ & $1.40 \times 10^{-31}$ \\
$(0.5,0.5)$ & $5.55 \times 10^{-17}$ & $3.57 \times 10^{-16}$ & $8.85 \times 10^{-28}$ & $1.00 \times 10^{-30}$ \\
$(0.6,0.6)$ & $5.55 \times 10^{-17}$ & $2.34 \times 10^{-16}$ & $8.88 \times 10^{-28}$ & $6.86 \times 10^{-31}$ \\
$(0.7,0.7)$ & $1.11 \times 10^{-16}$ & $1.54 \times 10^{-17}$ & $5.55 \times 10^{-17}$ & $5.55 \times 10^{-17}$ \\
$(0.8,0.8)$ & $1.67 \times 10^{-16}$ & $5.58 \times 10^{-17}$ & $5.55 \times 10^{-17}$ & $5.55 \times 10^{-17}$ \\
$(0.9,0.9)$ & $1.67 \times 10^{-16}$ & $2.11 \times 10^{-16}$ & $5.55 \times 10^{-17}$ & $5.55 \times 10^{-17}$ \\
\hline
\end{tabular}

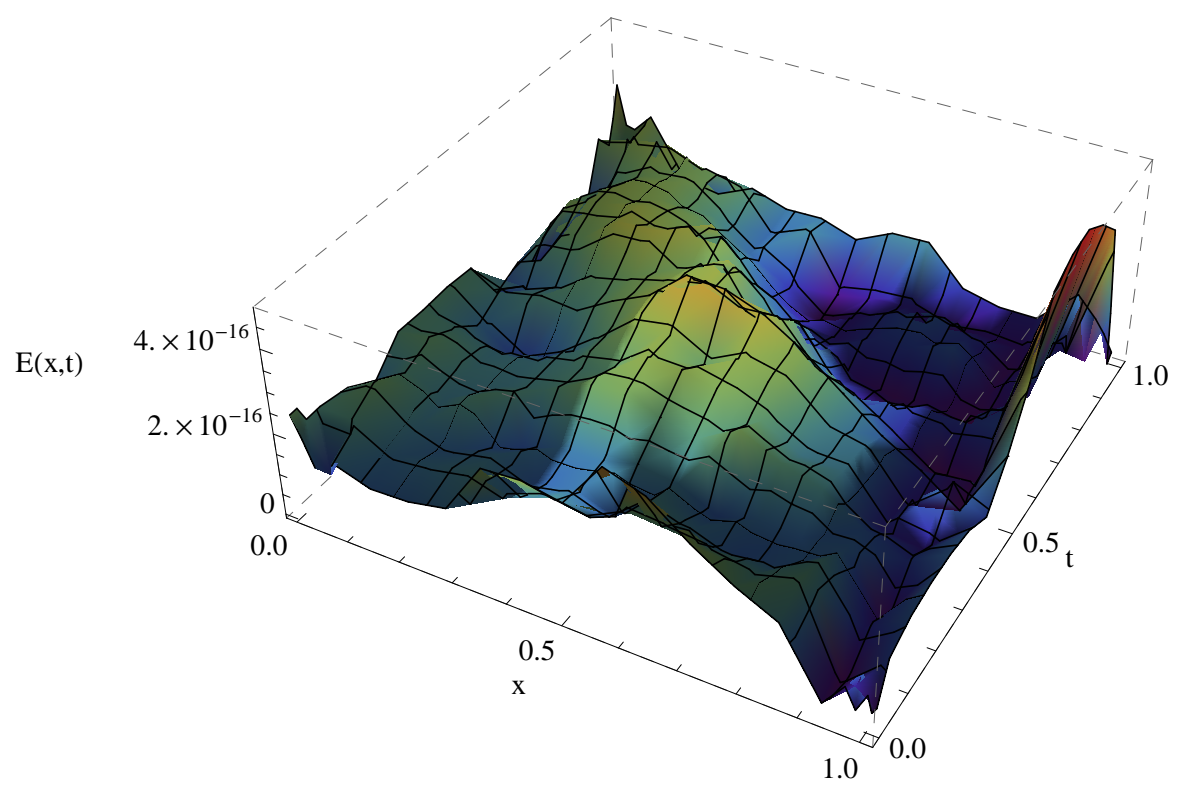

Figure 2. Space-graph of AEs related to Example 2 for $N=M=4$. 


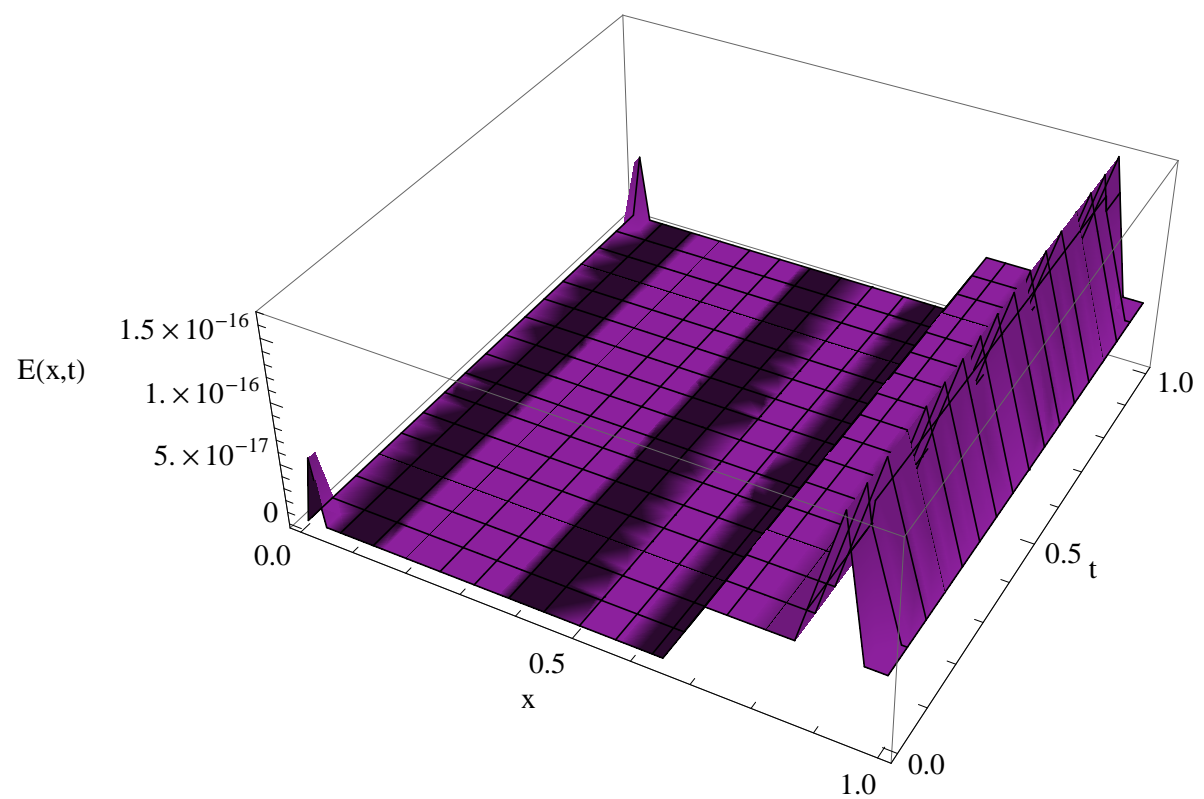

Figure 3. Space-graph of AEs related to Example 2 for $N=M=8$.

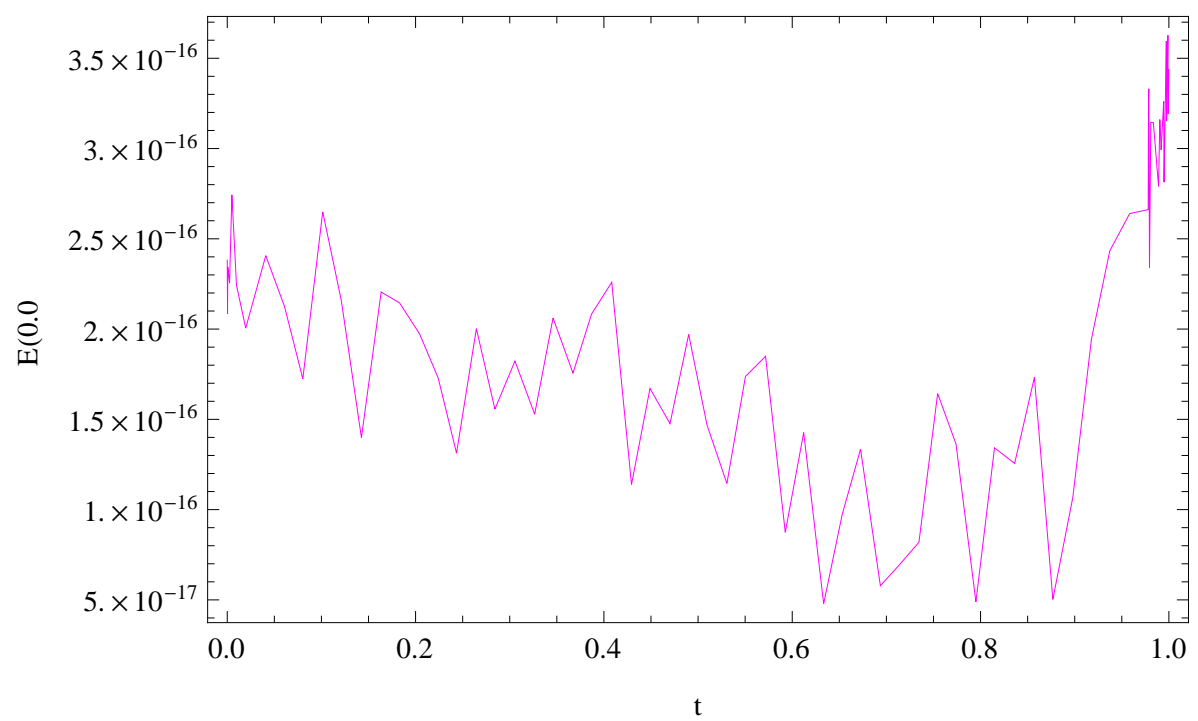

Figure 4. $t$-direction of absolute error of Example 2 at $x=0$ and $N=M=4$. 


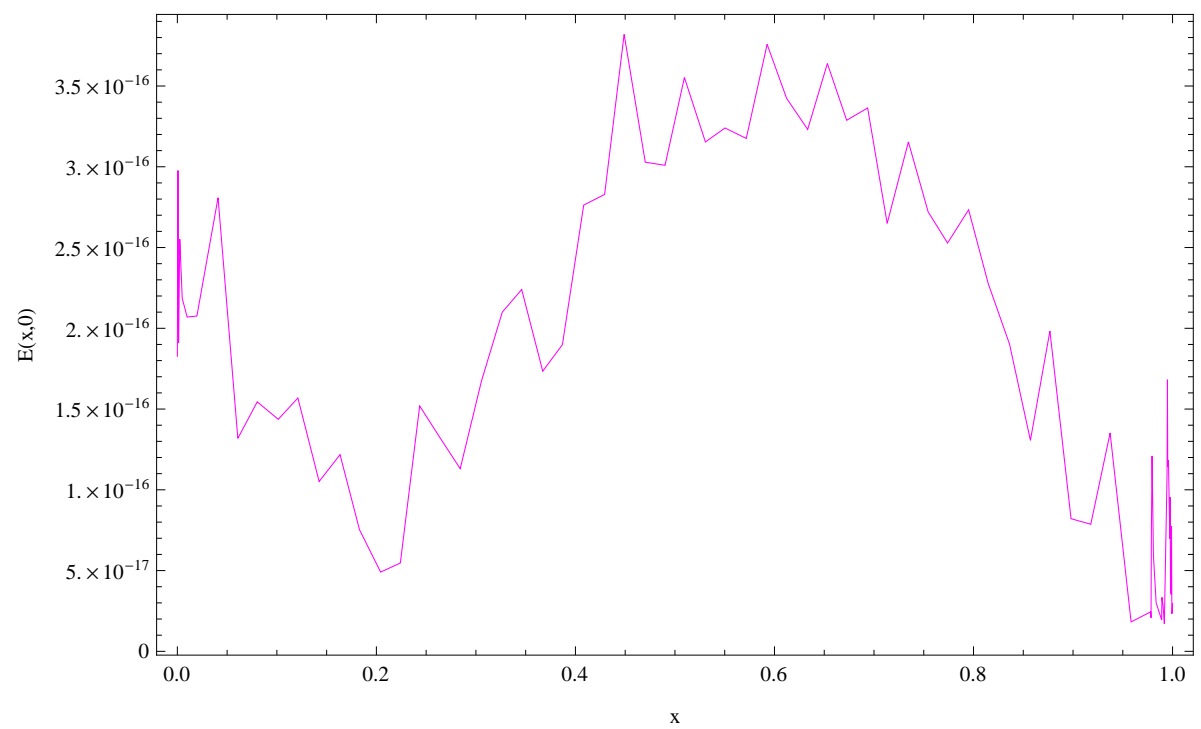

Figure 5. $x$-direction of absolute error of Example 2 at $t=0$ and $N=M=4$.

3. Example. We discuss the following problem

$$
u(x, t)=f(x, t)+\int_{0}^{x} \int_{0}^{t}(x-y)^{\frac{1}{4}}(t-\tau)^{\frac{-1}{4}} u(y, \tau) d \tau d y
$$

and $f(x, t)$ given by the exact solution $u(x, t)=x-t$.

The AEs for example 3 are listed in Table 3. The obtained results show that the proposed method is simple and very accurate numerical scheme for solving several mathematical physics problems.

Table 3. AEs for example 3.

\begin{tabular}{|c|c|c|c|c|}
\hline$(x, t)$ & $N=M=2$ & $N=M=4$ & $N=M=6$ & $N=M=8$ \\
\hline$(0,0)$ & $3.70 \times 10^{-17}$ & $1.85 \times 10^{-17}$ & $5.27 \times 10^{-30}$ & $1.03 \times 10^{-47}$ \\
$(0.1,0.1)$ & $1.67 \times 10^{-17}$ & $4.71 \times 10^{-17}$ & $2.18 \times 10^{-23}$ & $4.29 \times 10^{-40}$ \\
$(0.2,0.2)$ & $1.65 \times 10^{-17}$ & $3.88 \times 10^{-17}$ & $4.36 \times 10^{-23}$ & $8.58 \times 10^{-40}$ \\
$(0.3,0.3)$ & $4.89 \times 10^{-18}$ & $3.93 \times 10^{-17}$ & $6.54 \times 10^{-23}$ & $1.29 \times 10^{-39}$ \\
$(0.4,0.4)$ & $2.38 \times 10^{-17}$ & $5.45 \times 10^{-17}$ & $8.72 \times 10^{-23}$ & $1.72 \times 10^{-39}$ \\
$(0.5,0.5)$ & $2.62 \times 10^{-17}$ & $2.35 \times 10^{-17}$ & $1.09 \times 10^{-22}$ & $2.15 \times 10^{-39}$ \\
$(0.6,0.6)$ & $7.38 \times 10^{-18}$ & $3.14 \times 10^{-18}$ & $1.31 \times 10^{-22}$ & $2.57 \times 10^{-39}$ \\
$(0.7,0.7)$ & $2.79 \times 10^{-17}$ & $6.18 \times 10^{-17}$ & $1.53 \times 10^{-22}$ & $3.00 \times 10^{-39}$ \\
$(0.8,0.8)$ & $6.55 \times 10^{-17}$ & $1.98 \times 10^{-16}$ & $1.74 \times 10^{-22}$ & $3.43 \times 10^{-39}$ \\
$(0.9,0.9)$ & $8.18 \times 10^{-17}$ & $2.13 \times 10^{-16}$ & $1.96 \times 10^{-22}$ & $3.86 \times 10^{-39}$ \\
\hline
\end{tabular}




\section{Conclusion}

In this work, a spectral method for solving two-dimensional fractional integral equations with weakly singular based on left-sided mixed Riemann-Liouville integral of order $\nu$ were presented and developed. Using combination between shifted Legendre polynomials and shifted Chebyshev polynomial as basis functions of the spectral collocation method and the Gauss-quadrature formula, the two-dimensional fractional integral equations with weakly singular is reduced into a problem consisting of easily solvable system of algebraic equations. During three numerical applications, we ensure that the present method is simple and very accurate numerical scheme for solving several mathematical

physics problems. Very accurate numerical results are reported by using a limited number of nodes.

\section{References}

[1] L.E. Kosarev, Applications of integral equations of the first kind in experiment physics., Comput. Phys. Commun., 20 (1980) 69-75.

[2] H. Brunner, 1896-1996: One hundred years of Volterra integral equation of the first kind, Appl. Numer. Math., 24 (1997) 83-93.

[3] A. M. Wazwaz, Linear and Nonlinear Integral Equations Methods and Applications, Springer, Heidelberg, Dordrecht, London, New York, 2011.

[4] A. Wazwaz, A First Course in Integral Equations, World Scientific Publishing, Singapore, 1997.

[5] A. Wazwaz, A reliable modification of Adomian decomposition method, Appl. Math. Comput., 102 (1999) 77-86.

[6] A. D. Polyanin and A.V. Manzhirov, Handbook of Integral Equations, CRC Press, Boca Raton, 1998.

[7] R. Metzler and J. Klafter, The restaurant at the end of the random walk: Recent developments in the description of anomalous transport by fractional dynamics, J. Phys. A, 37 (2004) 161-208.

[8] R. T. Baillie, Long memory processes and fractional integration in econometrics, J. Econometrics, 73 (1996) 5-59.

[9] R. L. Magin, Fractional Calculus in Bioengineering, Begell House Publishers, 2006.

[10] D. A. Benson, S.W. Wheatcraft and M.M. Meerschaert, Application of a fractional advection-dispersion equation, Water Resour. Res., 36 (2000) 1403-1412.

[11] S. Abbas and M. Benchohra, Fractional order integral equations of two independent variables, Appl. Math. Comput., 227 (2014) 755-761.

[12] S. Abbas and M. Benchohra, Fractional Order Riemann-Liouville Integral Equations with Multiple Time Delays, Applied Mathematics, 12 (2012) 79-87.

[13] R. Hilfer, Applications of Fractional Calculus in Physics, World Scientific, Singapore, 2000.

[14] Y.L. Jiang and X.L Ding, Waveform relaxation methods for fractional differential equations with the Caputo derivatives, J. Comput. Appl. Math., 238 (2013) 51-67.

[15] A.A. Kilbas, H.M. Srivastava and J.J. Trujillo, Theory and Applications of Fractional Differential Equations, Elsevier, 2006.

[16] H. Wang and N. Du, Fast alternating-direction finite difference methods for threedimensional space-fractional diffusion equations, J. Comput. Phys., 258 (2014) 305-318.

[17] J. Liu and G. Hou, Numerical solutions of the space- and time-fractional coupled Burgers equation by generalized differential transform method, Appl. Math. Comput., 217 (2011) 7001-7008.

[18] L. Wang, Y. Ma and Z. Meng, Haar wavelet method for solving fractional partial differential equations numerically, Appl. Math. Comput., 227 (2014) 66-76

[19] R.K. Pandey, O.P. Singh and V.K. Baranwal, An analytic algorithm for the space-time fractional advection-dispersion equation, Comput. Phys. Commun. 182 (2011) 1134-1144. 
[20] I. Area, J. D. Djida, J. Losada and J.J. Nieto, On Fractional Orthonormal Polynomials of a Discrete Variable, Discrete Dynamics in Nature and Society 2015 (2015), Article ID 141325, 7 pages

[21] I. Podlubny, Fractional Differential Equations, Academic Press, San Diego, (1999).

[22] M.A. Abdelkawy, M.A. Zaky, A.H. Bhrawy and D. Baleanu, Numerical simulation of time variable fractional order mobile-immobile advection-dispersion model, Rom. Rep. Phys., 67 (3) 2015. frac

[23] A.H. Bhrawy and M.A. Zaky, Numerical simulation for two-dimensional variable-order fractional nonlinear cable equation, Nonlinear Dyn., 80(1) (2015) 101-116

[24] A.H. Bhrawy, Engy A. Ahmed and D. Baleanu, An efficient collocation technique for solving generalized Fokker-Planck type equations with variable coefficients, P. Rom. Acad. A, 15 (2014) 322-330

[25] W. Shaoa and X. Wu, An effective Chebyshev tau meshless domain decomposition method based on the integration-differentiation for solving fourth order equations, J. Comput. Phys., 231(2012) 7695-7714

[26] J. Chena, Y. Huanga, H. Ronga, T. Wub and T. Zen, A multiscale Galerkin method for second-order boundary value problems of Fredholm integro-differential equation, J. Comput. Appl. Math., 290 (2015) 633-640

[27] Y. Chen and J. Zhou, Error estimates of spectral Legendre-Galerkin methods for the fourthorder equation in one dimension, Appl. Math. Comput., 268 (2015) 1217-1226

[28] G. Kitzler and J. Schöberl, A high order space-momentum discontinuous Galerkin method for the Boltzmann equation, 70 (2015)1539-1554

[29] A.H. Bhrawy, An efficient Jacobi pseudospectral approximation for nonlinear complex generalized Zakharov system, Appl. Math. Comput., 247 (2014) 30-46.

[30] E.H. Doha, A.H. Bhrawy, M.A. Abdelkawy and R.A.V. Gorder, Jacobi-Gauss-Lobatto collocation method for the numerical solution of $1+1$ nonlinear Schrödinger equations, J. Comput. Phys., 261 (2014)244-255.

[31] E.H. Doha, A.H. Bhrawy and S.S. Ezz-Eldien, A new Jacobi operational matrix: An application for solving fractional differential equations, Appl. Math. Model., 36 (2012) 4931-4943.

[32] A.H. Bhrawy, E.H. Doha, D. Baleanu and S.S. Ezz-Eldien, A spectral tau algorithm based on Jacobi operational matrix for numerical solution of time fractional diffusion-wave equations, J. Comput. Phys.,293 (2015) 142-156.

[33] E.H. Doha, On the construction of recurrence relations for the expansion and connection coefficients in series of Jacobi polynomials, J. Phys. A Math. Gen. 37 (2004) 657-675.

[34] A.H. Bhrawy, M.A. Zaky and D. Baleanu, New numerical approximations for space-time fractional Burgers' equations via a Legendre spectral-collocation method, Rom. Rep. Phys., 67(2) (2015).

[35] A.H. Bhrawy, M. A. Abdelkawy, J. Tenreiro Machado and A. Z. M. Amin, Legender-GaussLobatto collocation method for solving multi-dimensional Fredholm integral equations., Comput. Math. Appl., Doi: 10.1016/j.camwa.2016.04.011

[36] M.R. Eslahchi, M. Dehghan and M. Parvizi, Application of the collocation method for solving nonlinear fractional integro-differential equations,J. Comput. Appl. Math., 257 (2014) 105-128.

[37] J. Shen, T. Tang and L. L. Wang, SPECTRAL METHODS Algorithms, Analyses and Applications, (Springer; 2011 edition August 31, 2011).

[38] C. Canuto, M. Y. Hussaini, A. Quarteroni and T.A. Zang, Spectral Methods: fundamentals in single domains, (Springer-Verlag, Berlin, Heidelberg, 2006).

[39] M. A. Abdelkawy, S. S. Ezz-Eldien and A. Z. M.Amin, A Jacobi Spectral Collocation Scheme for Solving Abel's Integral Equations, Progr. Fract. Differ. Appl.1, 3 (2015) 187200.

[40] A. H. Bhrawy, A.A. Al-Zahrani, Y.A. Alhamed and D. Baleanu, A new generalized Laguerre-Gauss collocation scheme for numerical solution of generalized fractional Pantograph equations, Rom. J. Phys., 59 (2014) 646-657

[41] S. R. Lau and R. H. Price, Sparse spectral-tau method for the three-dimensional helically reduced wave equation on two-center domains, J. Comput. Phys., 231(2012) 7695-7714 
[42] F. Ghoreishi and S. Yazdani, An extension of the spectral Tau method for numerical solution of multi-order fractional differential equations with convergence analysis, Comput. Math. Appl., 61 (2011) 30-43

[43] E. H. Doha and A. H. Bhrawy, An efficient direct solver for multidimensional elliptic Robin boundary value problems using a Legendre spectral-Galerkin method, Comput. Math. Appl., 64 (2012) 558-571

[44] Y. Luke, The Special Functions and Their Approximations, vol. 2, Academic Press, New York, (1969).

[45] E. H. Doha, A. H. Bhrawy and R. M. Hafez, A Jacobi-Jacobi dual-Petrov-Galerkin method for third- and fifth-order differential equations, Math. Comput. Model, 53 (2011) 18201832.

[46] I. Area, D.K. Dimitrov, E. Godoy, V.G. Paschoa, Approximate Calculation of Sums I: Bounds for the Zeros of Gram Polynomials, SIAM J. Numer. Anal., 54(4), (2014)22102227.

[47] I. Area, D.K. Dimitrov, E. Godoy and V.G. Paschoa, Approximate Calculation of Sums II: Gaussian Type Quadrature, SIAM J. Numer. Anal., 52(4), (2016)1867-1886.

[48] E.H. Doha and A.H. Bhrawy, An efficient direct solver for multidimensional elliptic Robin boundary value problems using a Legendre spectral-Galerkin method, Computers and Mathematics with Applications 64 (2012) 558-571.

[49] E. Godoy, A. Ronveaux, A. Zarzo and I. Area, Minimal recurrence relations for connection coefficients between classical orthogonal polynomials: Continuous case, Journal of Computational and Applied Mathematics 84 (1997) 257-275.

[50] A. Ronveaux, A. Zarzo, I. Area and E. Godoy, Transverse limits in the Askey tableau, Journal of Computational and Applied Mathematics 99 (1998) 327-335. 